NISTIR 7526

\title{
Determination of Wave Motion Correction Values Required for Comparison Calibrations of a New Working Standard Microphone
}

\author{
Randall P. Wagner \\ Victor Nedzelnitsky
}

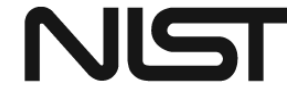

National Institute of Standards and Technology 
NISTIR 7526

\title{
Determination of Wave Motion Correction Values Required for Comparison Calibrations of a New Working Standard Microphone
}

\author{
Randall P. Wagner \\ Victor Nedzelnitsky \\ Sensor Development \& Application Group \\ Manufacturing Metrology Division \\ Manufacturing Engineering Laboratory \\ National Institute of Standards and Technology
}

November 2008

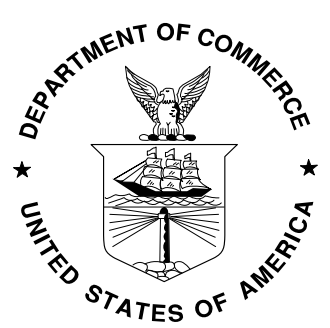

U.S. Department of Commerce

Carlos M. Gutierrez, Secretary

National Institute of Standards and Technology

Patrick D. Gallagher, Deputy Director 


\title{
Determination of Wave Motion Correction Values Required for Comparison Calibrations of a New Working Standard Microphone
}

\author{
Randall P. Wagner and Victor Nedzelnitsky \\ National Institute of Standards and Technology, \\ Gaithersburg, MD 20899-8220
}

Comparison calibrations of microphones based on the reciprocity method are performed with both the microphone under test and a reference standard microphone inserted into an acoustic coupler. For these calibrations, the wave motion correction $W$ is needed to account for the departure from spatially uniform sound pressure amplitude in the coupler at frequencies for which the coupler interior dimensions are not small compared to a wavelength of sound. This internal report describes the procedure used to determine the values of $W_{4192}$ applicable to comparison calibrations of a relatively new working standard microphone, the Bruel and Kjaer model $4192^{1}$, and provides these values.

Key words: acoustic calibration; calibration of microphones; microphone calibration; NIST microphone calibration; reciprocity calibration; standard microphone; wave motion correction; working standard microphone.

\footnotetext{
${ }^{1}$ Certain commercial equipment, instruments, or materials are identified in this paper in order to specify the experimental procedure adequately. Such identification is not intended to imply recommendation or endorsement by the National Institute of Standards and Technology, nor is it intended to imply that the materials or equipment identified are necessarily the best available for the purpose.
} 


\section{TABLE OF CONTENTS}

Page

1. INTRODUCTION/BACKGROUND .

Fig. 1. Sectional view of reference standard microphone and test microphone inserted into an acoustic coupler for a reciprocity-based comparison calibration of the test microphone.

2. EXPERIMENTAL SETUP

Fig. 2. Block diagram of the system used to measure the voltage ratio $U_{2} / U_{1}$ of receiver voltage to transmitter voltage.

3. Procedures for Acquiring Wave Motion Correction Data

Table 1a. List of test frequencies at which measurements were made in the $4 \mathrm{~cm}^{3}$ coupler to determine values of $W_{4192}$, which are values of the wave motion correction applicable to reciprocitybased comparison calibrations of a Bruel and Kjaer model 4192 microphone.

Table 1b. List of test frequencies at which measurements were made in the $20 \mathrm{~cm}^{3}$ coupler to determine values of $W_{4192}$, which are values of the wave motion correction applicable to reciprocitybased comparison calibrations of a Bruel and Kjaer model 4192 microphone.

\section{DATA REDUCTION TO ObTAin WAVE Motion CORRECTION VALUES AT CALIBRATION FREQUENCIES IN HYDROGEN-FILLED COUPLERS}

5. RESULTS AND SUMMARY

Table 2. Values of the wave motion correction $W_{4192}$, and $20 \log W_{4192}$, applicable to the NIST fixed-cost comparison calibrations of a Bruel and Kjaer model 4192 microphone performed with the hydrogen-filled $20 \mathrm{~cm}^{3}$ coupler.

Table 3. Values of the wave motion correction $W_{4192}$, and $20 \log W_{4192,}$ applicable to the NIST fixed-cost comparison calibrations of a Bruel and Kjaer model 4192 microphone performed with the hydrogen-filled $4 \mathrm{~cm}^{3}$ coupler..

6. UnCERTAinties OF WAVE Motion CORRECTION MEASUREMENT RESUlTS

Table 4. Summary of uncertainties of the measured wave motion correction values 12

7. REFERENCES 


\section{INTRODUCTION/BACKGROUND}

The National Institute of Standards and Technology (NIST) fixed-cost acoustic measurement services SP25010C through SP25040C [1] determine the pressure sensitivity level of a customer's microphone (the test microphone) by using a reciprocity-based method for comparison calibration with reference standard microphones. Each such reference standard microphone is a NIST-owned International Electrotechnical Commission (IEC) and American National Standards Institute (ANSI) Type LS1Pn laboratory standard microphone $[2,3]$ that has been calibrated by the reciprocity method [4-6] to determine both its sensitivity and its driving-point electrical impedance, which are used in the comparison calibration. During this comparison calibration, the reference standard microphone and the test microphone are inserted into ports on opposite sides of an acoustic coupler, which has an internal cavity of known volume between the microphone diaphragms, as shown in Fig. 1. The reference standard microphone is electrically driven and used as a sound source, called the transmitter (microphone 1), and the test microphone is used as a receiver (microphone 2), which produces an output voltage in response to the sound pressure at its diaphragm in the coupler. The ratio of open-circuit voltage $U_{2}$ at the output of the test microphone, to the voltage $U_{1}$ driving the reference standard microphone is measured. At each frequency of calibration, measurement results are combined with the acoustic transfer impedance calculated for the gas filling the coupler cavity. This transfer impedance calculation is based on the model of an acoustic compliance, using the equivalent volumes of the microphones, the geometric volume of the coupler cavity including the front cavity volumes of the microphones, and the properties of the gas filling the coupler. The other parameters that are included are the calculated frequency-dependent corrections to this impedance for the effects of heat conduction at the interior boundaries of the coupler with microphones, and for the effects of acoustic wave motion. The wave motion correction $W$ is needed to account for the departure from a spatially uniform sound pressure amplitude in the coupler at frequencies for which the coupler interior dimensions are not small compared to a wavelength of sound. To cover the entire frequency range of the NIST measurement services, couplers of different sizes are used and are filled with air or hydrogen, as appropriate, for the measurement frequency range and coupler size. Hydrogen is used as a fill gas at high frequencies because the larger speed of sound in hydrogen than in air results in a longer wavelength at a given frequency. The upper frequency limit of a given coupler is extended because of the increase in wavelength when the coupler is filled with hydrogen rather than air.

At each frequency of calibration, the calculation procedure uses a parameter $B$ (in decibels) that contains the effect on the test microphone sensitivity level of all other parameters except the voltage ratio and static ambient (barometric) pressure data. The test microphone sensitivity level is determined from $B$ and the measured data for the voltage ratio and barometric pressure. For a frequently used test configuration in which both the reference standard and test microphones are Bruel and Kjaer model $4160^{1}$ microphones, $B$ is denoted

\footnotetext{
${ }^{1}$ Certain commercial equipment, instruments, or materials are identified in this paper in order to specify the experimental procedure adequately. Such identification is not intended to imply recommendation or endorsement by the National Institute of Standards and Technology, nor is it intended to imply that the materials or equipment identified are necessarily the best available for the purpose.
} 
Fig. 1. Sectional view of reference standard microphone and test microphone inserted into an acoustic coupler for a reciprocity-based comparison calibration of the test microphone.

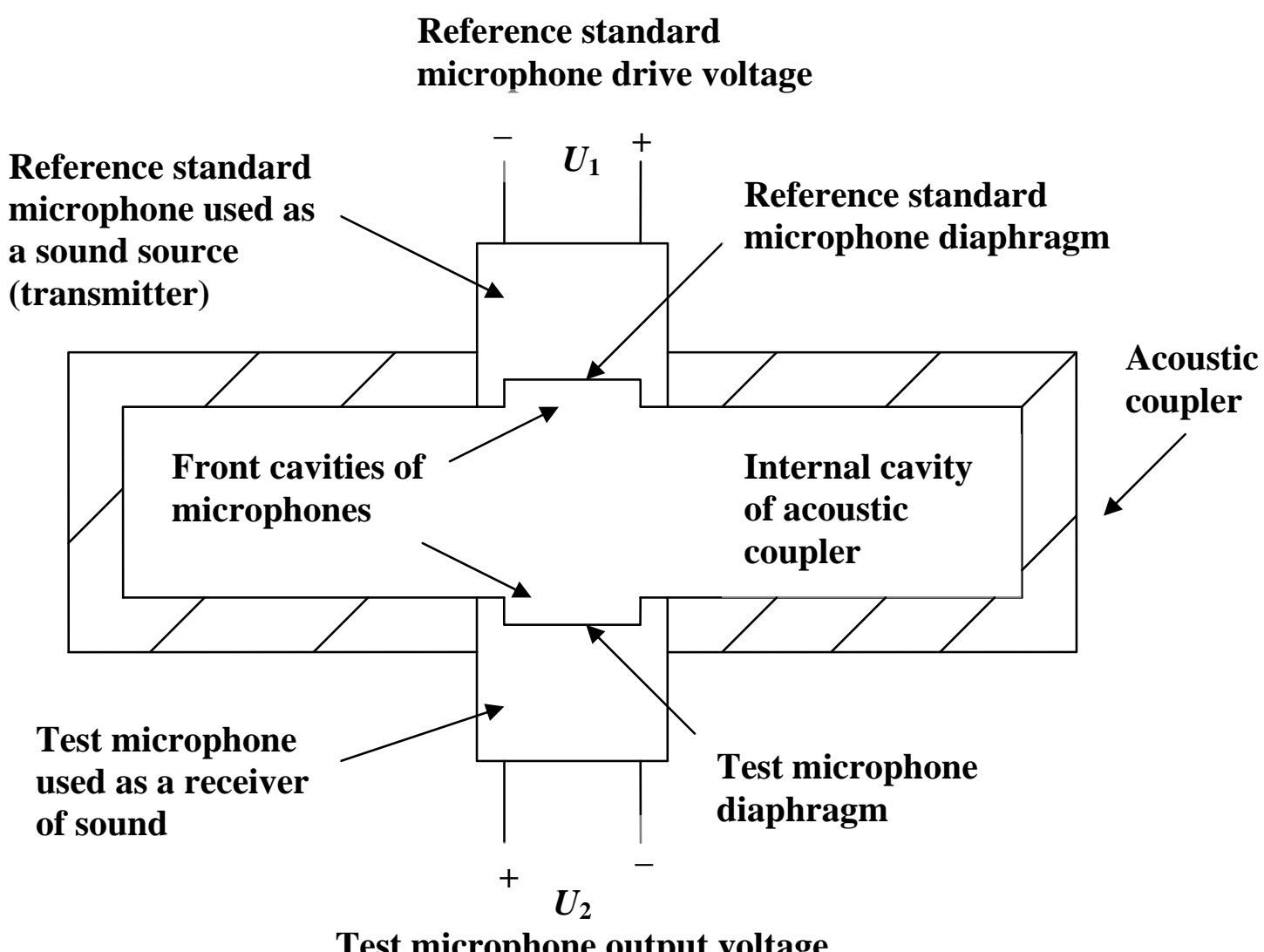

Test microphone output voltage 
as $B_{4160}$, and values of $B_{4160}$ are used in this calculation procedure. The Bruel and Kjaer model 4160 microphones are of IEC and ANSI Type LS1Pn. For other test microphones, such as IEC and ANSI Type LS2aP microphones [2,3], and a number of manufacturers' models of IEC Type WS2 microphones [7], modifications are applied to the values of $B_{4160}$ to account for the differences in the test microphone characteristics.

In order to calibrate a relatively new model of IEC Type WS2 microphone, the Bruel and Kjaer model 4192, it was necessary to determine the values of the frequency-dependent correction $B_{\text {cor,4192. }}$. This correction is applicable to the comparison calibration of a Bruel and Kjaer model 4192 test microphone with a Bruel and Kjaer model 4160 reference standard microphone and is used to modify the values of $B_{4160}$. The geometrical configuration of the model 4192 microphone differs slightly from that of the other IEC Type WS2 microphones

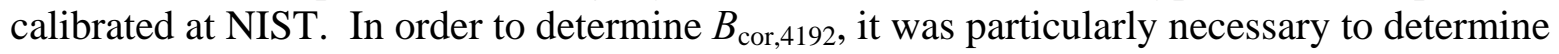
experimentally the wave motion correction $W_{4192}$ for the couplers configured with a Bruel and Kjaer model 4192 test microphone and a Bruel and Kjaer model 4160 reference standard microphone. This internal report describes the procedure used to determine the values of $W_{4192}$, and provides these values.

\section{EXPERIMENTAL SETUP}

The experimental setup used to perform the measurements required to determine the values of $W_{4192}$ is the same setup currently used to perform reciprocity-based comparison calibrations of microphones for NIST customers. This setup consists of one of two cylindrical couplers designed to accommodate the transmitter-receiver pair of microphones, a system for measuring the voltage ratio $U_{2} / U_{1}$ at each frequency, and a hydrogen supply and retention system for filling the couplers with hydrogen. The gas grade purity of the hydrogen used is Matheson Purity (99.9995 \%) supplied by Matheson Tri-Gas. Equipment for measuring the ambient environmental conditions, particularly the barometric pressure, is also included.

One acoustic coupler has a nominal cavity volume of $20 \mathrm{~cm}^{3}$. For customer calibrations, this coupler is air-filled for frequencies from $50 \mathrm{~Hz}$ to $700 \mathrm{~Hz}$, and hydrogenfilled for frequencies from $1 \mathrm{kHz}$ to $10 \mathrm{kHz}$. A smaller coupler, which has a nominal cavity volume of $4 \mathrm{~cm}^{3}$, is hydrogen-filled for calibrations at frequencies from $11 \mathrm{kHz}$ to $20 \mathrm{kHz}$. Each coupler includes a pair of small-diameter capillary tubes connecting the interior volume of the coupler to the ambient air. Such capillary tubes are necessary to prevent the unwanted effects of changes in ambient barometric pressure. These effects would produce differences between the static pressures within the coupler and within the back-cavity volumes of the microphones, which are vented to ambient pressure through equalization vents on the sides of each microphone. Differences in static pressure between the exterior and interior surfaces of the microphone diaphragms would apply a static force to the diaphragms of the microphones and mechanically "bias" them to abnormal sensitivities in response to sound pressure. These couplers are filled with hydrogen by using the apparatus described in Ref. [8] connected to both capillary tubes in the case of the $4 \mathrm{~cm}^{3}$ coupler, or only one such tube in the case of the $20 \mathrm{~cm}^{3}$ coupler. 
Both couplers were constructed with ports that readily accept IEC and ANSI Type LS1Pn microphones without adapters. IEC Type WS2 microphones fit into the couplers by means of an adapter having annulus and front-cavity geometries similar to that of an IEC and ANSI Type LS1Pn microphone. This adapter uses an O-ring of synthetic rubber with petroleum jelly to provide a seal at the outer diameter of the microphone, so that only the diaphragm is exposed to the sound field within the coupler cavity. With this adapter, it is possible to seal the cavity sufficiently well so that hydrogen can be used in the same frequency ranges as for the IEC and ANSI Type LS1Pn microphone calibrations.

A block diagram of the system used to measure $U_{2} / U_{1}$ is shown in Fig. 2. To determine this ratio at a given frequency, the sound/attenuator switch is first set to the sound position. This setting causes the signal generator output to drive the transmitter through the transmitter drive unit, producing a sound pressure in the coupler. This sound pressure produces an output voltage signal from the receiver, which is connected to an insert voltage preamplifier. At the output of this preamplifier, the signal is routed through a measuring amplifier and band-pass filter. The amplified and filtered signal voltage produces a reading on an expanded scale meter capable of resolving $0.01 \mathrm{~dB}$ increments in voltage level. The meter reading is noted since it must be matched during the next step of the measurement. Without changing any settings of the signal generator, amplifier, and filter, the sound/attenuator switch is set to the attenuator position. This setting disconnects the signal generator output from the transmitter and grounds the circuit point at which the generator had been connected so that the transmitter produces essentially no sound in the coupler. Furthermore, this switch setting connects the signal generator output to the input of an attenuation network that consists of a variable attenuator and resistors that provide additional, fixed attenuation. The signal is electrically applied through the attenuation network and the insert voltage preamplifier so that the output of the network acts as a voltage source in series with the electrical terminals of the receiver. Following the receiver terminals, the rest of the signal path remains unchanged. The voltage supplied by the network is amplified, filtered, and produces a meter reading just as the voltage at the output of the receiver had with acoustic excitation when the sound/attenuator switch was in the sound position. The variable attenuator is adjusted until the reading observed on the meter is the same as the reading noted when the switch had been in the sound position. The sound/attenuator switch is then set back to the sound position to check whether the meter indication is still the same. The value of attenuation to which the variable attenuator is adjusted is then added to the total value of the additional fixed attenuation to obtain the total attenuation $A$. The measured value of $U_{2} / U_{1}$ is found from $A$ using Eq. (1).

$$
U_{2} / U_{1}=\operatorname{antilog}\left(\frac{-A}{20}\right)
$$

Voltage ratio data were acquired for microphone pairs formed by using one of two Bruel and Kjaer model 4160 microphones as the transmitter with one of four Bruel and Kjaer model 4192 microphones as the receiver. These two Bruel and Kjaer model 4160 microphones are periodically calibrated at NIST by the reciprocity method for use as reference standard microphones in reciprocity-based comparison calibrations of customer microphones. Combining these microphones in this manner produced eight transmitterreceiver pairs for acquisition of the data required to determine the values of $W_{4192}$. 
Fig. 2. Block diagram of the system used to measure the voltage ratio $U_{2} / U_{1}$ of receiver voltage to transmitter voltage.

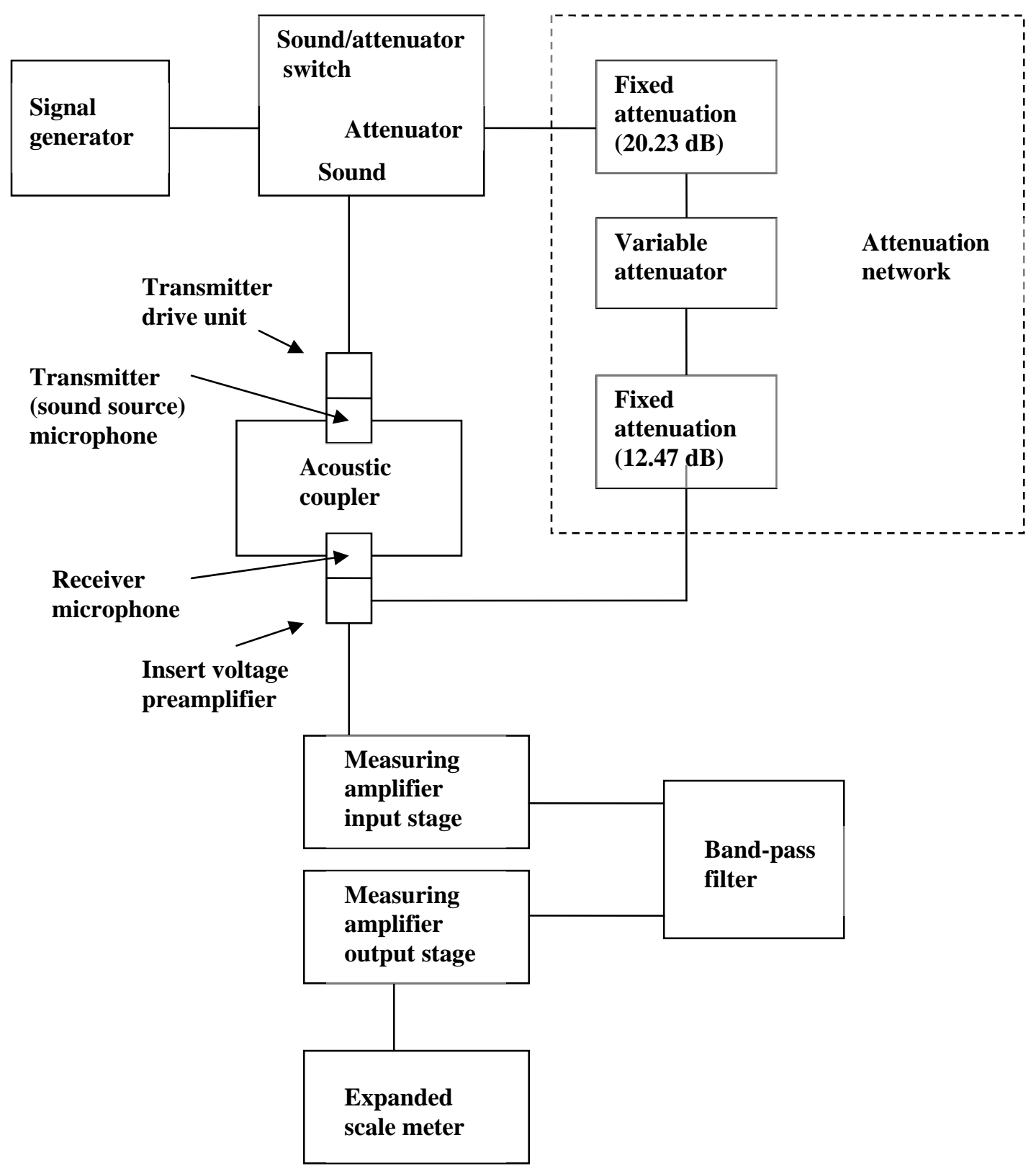




\section{Procedures for Acquiring Wave Motion Correction Data}

To set up the microphones with the $4 \mathrm{~cm}^{3}$ coupler for voltage ratio measurements, a Bruel and Kjaer model 4192 microphone was threaded into the adapter used for IEC Type WS2 microphones after petroleum jelly was applied to the O-ring in this adapter. The adapter annulus, which had a thin layer of petroleum jelly applied on its surface, was placed in contact with one of the plane mating surfaces of the air-filled coupler. One of the Bruel and Kjaer model 4160 reference standard microphones, which had a thin layer of petroleum jelly applied on its front annulus, was then placed on the opposite side of the coupler so that the annulus was in contact with the other plane mating surface of the coupler.

Measurements of $U_{2} / U_{1}$ were made for a given pair of microphones at selected test frequencies in the air-filled coupler by adjusting the variable attenuator to match the attenuated insert voltage to the output voltage produced with acoustic excitation of the receiver. Table 1a lists the selected test frequencies for the $4 \mathrm{~cm}^{3}$ coupler, and Table $1 \mathrm{~b}$ lists the selected test frequencies for the $20 \mathrm{~cm}^{3}$ coupler. These frequencies were determined by dividing the midpoint frequencies between each pair of adjacent frequencies used for customer calibrations by the ratio of the second radial mode resonance frequency $f_{\mathrm{r}}^{\mathrm{h}}$ of the hydrogen-filled coupler cavity to the second radial mode resonance frequency $f_{\mathrm{r}}^{\mathrm{a}}$ of the airfilled coupler cavity. A working value of $f_{\mathrm{r}}^{\mathrm{h}} / f_{\mathrm{r}}^{\mathrm{a}}$ had been determined from preliminary measurements for each coupler. This value was 3.60 for the $20 \mathrm{~cm}^{3}$ coupler, and 3.69 for the $4 \mathrm{~cm}^{3}$ coupler. Ideally, this value should be about 3.87, which is the ratio of the speed of sound in hydrogen to the speed of sound in air. However, a value of 3.87 is not achieved in practice due to impurities in the hydrogen, water vapor, and contamination due to residual air trapped in the system [6]. Additional test frequencies were included above and below the calculated range of test frequencies to determine the values of $W_{4192}$ applicable to the upper and lower calibration frequencies for both couplers. Measurements were performed in descending order of frequency starting at the highest frequency. The barometric pressure, temperature, relative humidity, and $f_{\mathrm{r}}^{\mathrm{a}}$ were measured immediately before and after the measurements of $U_{2} / U_{1}$.

After the air-filled coupler measurements were completed, the coupler was filled with hydrogen. In the case of the $4 \mathrm{~cm}^{3}$ coupler, the coupler was filled with hydrogen using the procedure described in Ref. [8], and $f_{\mathrm{r}}^{\mathrm{h}}$ was monitored. The value of $f_{\mathrm{r}}^{\mathrm{h}}$ was measured and recorded when it stopped increasing, and the hydrogen flow was turned off. Measurements of $U_{2} / U_{1}$ were then performed for the same microphone pair at the same test frequencies used for the measurements done in the coupler when filled with air. These measurements were also performed in descending order of frequency starting at the highest frequency. After $U_{2} / U_{1}$ was measured at the lowest frequency, the measurement of $U_{2} / U_{1}$ was repeated at the highest frequency. This value of $U_{2} / U_{1}$ was always within $\pm 0.01 \mathrm{~dB}$ of the value initially measured at the highest frequency. This result is acceptable because the resolution of the measurement of $U_{2} / U_{1}$ is $0.01 \mathrm{~dB}$. At that time, the measurement of $f_{\mathrm{r}}^{\mathrm{h}}$ was also repeated. As was done with the air-filled coupler measurements, the barometric pressure, temperature, and relative humidity were measured immediately before and after the measurements of $U_{2} / U_{1}$. 
Table 1a. List of test frequencies at which measurements were made in the $4 \mathrm{~cm}^{3}$ coupler to determine values of $W_{4192}$, which are values of the wave motion correction applicable to reciprocity-based comparison calibrations of a Bruel and Kjaer model 4192 microphone.

\begin{tabular}{|c|}
\hline Test frequencies $(\mathrm{Hz})$ for $4 \mathrm{~cm}^{3}$ coupler \\
\hline 5556 \\
\hline 5285 \\
\hline 5014 \\
\hline 4743 \\
\hline 4472 \\
\hline 4201 \\
\hline 3930 \\
\hline 3659 \\
\hline 3388 \\
\hline 3117 \\
\hline 2846 \\
\hline 2575 \\
\hline
\end{tabular}

Table 1b. List of test frequencies at which measurements were made in the $20 \mathrm{~cm}^{3}$ coupler to determine values of $W_{4192}$, which are values of the wave motion correction applicable to reciprocity-based comparison calibrations of a Bruel and Kjaer model 4192 microphone.

\begin{tabular}{|c|}
\hline Test frequencies $(\mathrm{Hz})$ for $20 \mathrm{~cm}^{3}$ coupler \\
\hline 2917 \\
\hline 2639 \\
\hline 2361 \\
\hline 2083 \\
\hline 1806 \\
\hline 1528 \\
\hline 1250 \\
\hline 972 \\
\hline 764 \\
\hline 625 \\
\hline 486 \\
\hline 347 \\
\hline 208 \\
\hline
\end{tabular}


After completing both the air-filled coupler and hydrogen-filled coupler sets of voltage ratio measurements using the $4 \mathrm{~cm}^{3}$ coupler at the test frequencies listed in Table 1a, measurements were made using the $20 \mathrm{~cm}^{3}$ coupler at the test frequencies listed in Table $1 \mathrm{~b}$ using the same procedures except for the hydrogen fill. This coupler was filled by connecting a single capillary tube, which was used as a gas inlet, to the hydrogen supply and retention system. When $f_{\mathrm{r}}^{\mathrm{h}}$ stopped increasing, the hydrogen supply was turned off by closing a single valve in the hydrogen supply and retention system, and then the measurements were performed.

The entire measurement process in both couplers was repeated with each of the other seven microphone pairs formed by using one of the Bruel and Kjaer model 4160 reference standard microphones as the transmitter and a Bruel and Kjaer model 4192 microphone as the receiver.

\section{Data Reduction to Obtain Wave Motion Correction Values at CALIBRATION FREQUENCIES IN HYDROGEN-FILLED COUPLERS}

At each test frequency, the value of $W_{4192}$ was calculated using

$$
W_{4192}=\frac{\left(U_{2} / U_{1}\right)^{\mathrm{h}}\left[\left(\frac{V_{\mathrm{g}} \Delta_{\mathrm{H}}^{\mathrm{h}}}{\kappa^{\mathrm{h}} P^{\mathrm{h}}}\right)+\left(\frac{V_{\mathrm{e}, 1}^{\mathrm{h}}}{\kappa^{\mathrm{h}} P_{\mathrm{r}}}\right)+\left(\frac{V_{\mathrm{e}, 2}^{\mathrm{h}}}{\kappa^{\mathrm{h}} P_{\mathrm{r}}}\right)\right]}{\left(U_{2} / U_{1}\right)^{\mathrm{a}}\left[\left(\frac{V_{\mathrm{g}} \Delta_{\mathrm{H}}^{\mathrm{a}}}{\kappa^{\mathrm{a}} P^{\mathrm{a}}}\right)+\left(\frac{V_{\mathrm{e}, 1}^{\mathrm{a}}}{\kappa^{\mathrm{a}} P_{\mathrm{r}}}\right)+\left(\frac{V_{\mathrm{e}, 2}^{\mathrm{a}}}{\kappa^{\mathrm{a}} P_{\mathrm{r}}}\right)\right]}
$$

where $V_{\mathrm{g}}$ is the total geometric volume in cubic meters of the cavity, $V_{\mathrm{e}, 1}$ and $V_{\mathrm{e}, 2}$ are the equivalent volumes in cubic meters of the Bruel and Kjaer model 4160 transmitter and the Bruel and Kjaer model 4192 receiver respectively, $P$ is the measured barometric pressure in pascals, $P_{\mathrm{r}}$ is the reference barometric pressure $(101.325 \mathrm{kPa})$ in pascals, $\kappa$ is the dimensionless ratio of specific heats for the gas in the cavity, and $\Delta_{\mathrm{H}}$ is the heat conduction correction (dimensionless) calculated according to Ref. [4]. The superscripts $h$ and a indicate whether the parameter is applicable to a hydrogen-filled or air-filled cavity respectively. The volume $V_{\mathrm{g}}$ is the sum of the coupler cavity volume, the geometric front cavity volume of the transmitter, and the geometric front cavity volume of the adapter into which the receiver has been threaded. This equation was derived from the expression for the product of the sensitivities for the transmitter and receiver given in Ref. [4]. Values of $\kappa^{\mathrm{a}}(1.402)$ and $\kappa^{\mathrm{h}}$ (1.405), which historically have been used at NIST, are given in Ref. [6]. No capillary tube correction was applied, since previous measurements indicated that this correction is negligible for the capillary tubes used.

To determine the frequency at which the values of $W_{4192}$ are applicable to measurements made in a hydrogen-filled coupler, the test frequency was scaled by the measured value of the ratio $f_{\mathrm{r}}^{\mathrm{h}} / f_{\mathrm{r}}^{\mathrm{a}}$. For each set of measurement data acquired with a given pair of microphones and a given gas in the coupler, the resonance frequency used to calculate 
this ratio was determined by averaging the resonance frequencies measured at the start and stop of data acquisition for that pair and gas.

The value of $W_{4192}$ applicable to a given frequency used for customer calibrations in a hydrogen-filled coupler was determined by linear interpolation using the wave motion correction values applicable to the two scaled frequencies between which the calibration frequency lies.

For each customer calibration frequency and coupler, the average value of $W_{4192}$ and the population standard deviation were calculated from the values of $W_{4192}$ determined for each of the eight microphone pairs.

\section{RESUlTS AND SUMMARY}

Values of $W_{4192}$, which are applicable to NIST fixed-cost comparison calibrations of Bruel and Kjaer model 4192 customer microphones performed with a Bruel and Kjaer model 4160 transmitter, are given in Table 2 and Table 3. Table 2 gives values applicable to such calibrations performed in the hydrogen-filled $20 \mathrm{~cm}^{3}$ coupler, and Table 3 gives values applicable to such calibrations performed in the hydrogen-filled $4 \mathrm{~cm}^{3}$ coupler.

The frequency-dependent values of $W_{4192}$ in Table 2 and Table 3 determined from the measurement data were used to calculate the values of the frequency-dependent correction

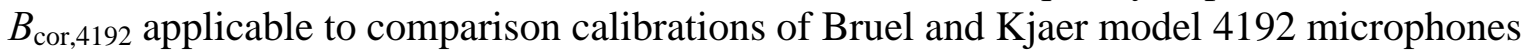
with the Bruel and Kjaer model 4160 reference standard microphones. Values of $B_{\text {cor,4192 }}$ were determined at each frequency of calibration applicable to the hydrogen-filled couplers using

$$
B_{\text {cor }, 4192}=20 \log \left[\frac{V_{\mathrm{g}} \Delta_{\mathrm{H}}^{\mathrm{h}}+V_{\mathrm{e}, 1}^{\mathrm{h}}+V_{\mathrm{e}, 2}^{\mathrm{h}}}{V_{\mathrm{g}} \Delta_{\mathrm{H}}^{\mathrm{h}}+2 V_{\mathrm{e}, 1}^{\mathrm{h}}}\right]+20 \log W_{4192}-20 \log W_{4160}
$$

where $W_{4160}$ is the wave motion correction applicable to the configuration with a Bruel and Kjaer model 4160 transmitter $\underline{\text { and }}$ receiver. When a Bruel and Kjaer model 4192 microphone is calibrated by reciprocity-based comparison with the Bruel and Kjaer model 4160 reference standard microphones, the value of $B_{\text {cor,4192 }}$ is added to the value of $B_{4160}$ to determine the sensitivity level of the Bruel and Kjaer model 4192 microphone. 
Table 2. Values of the wave motion correction $W_{4192}$, and $20 \log W_{4192}$, applicable to the NIST fixed-cost comparison calibrations of a Bruel and Kjaer model 4192 microphone performed with the hydrogen-filled $20 \mathrm{~cm}^{3}$ coupler.

\begin{tabular}{|r|r|r|r|}
\hline $\begin{array}{l}\text { Calibration } \\
\text { frequency (Hz) }\end{array}$ & $\begin{array}{l}\text { Wave motion } \\
\text { correction } W_{4192} \\
\text { (dimensionless) }\end{array}$ & $\begin{array}{l}\text { 20 log } W_{4192} \\
\text { (decibels) }\end{array}$ & $\begin{array}{l}\text { Expanded (coverage } \\
\text { factor k=2) } \\
\text { uncertainty (decibels) }\end{array}$ \\
\hline 10000 & 0.9874 & -0.110 & 0.030 \\
\hline 9000 & 0.9874 & -0.110 & 0.026 \\
\hline 8000 & 0.9881 & -0.104 & 0.023 \\
\hline 7000 & 0.9897 & -0.090 & 0.023 \\
\hline 6000 & 0.9917 & -0.072 & 0.023 \\
\hline 5000 & 0.9946 & -0.047 & 0.023 \\
\hline 4000 & 0.9971 & -0.025 & 0.021 \\
\hline 3000 & 0.9989 & -0.010 & 0.021 \\
\hline 2500 & 0.9996 & -0.003 & 0.021 \\
\hline 2000 & 0.9998 & -0.002 & 0.023 \\
\hline 1500 & 0.9997 & -0.003 & 0.022 \\
\hline 1000 & 0.9995 & -0.004 & 0.027 \\
\hline
\end{tabular}

Table 3. Values of the wave motion correction $W_{4192}$, and $20 \log W_{4192,}$, applicable to the NIST fixed-cost comparison calibrations of a Bruel and Kjaer model 4192 microphone performed with the hydrogen-filled $4 \mathrm{~cm}^{3}$ coupler.

\begin{tabular}{|r|r|l|r|}
\hline $\begin{array}{l}\text { Calibration } \\
\text { frequency (Hz) }\end{array}$ & $\begin{array}{l}\text { Wave motion } \\
\text { correction } W_{4192} \\
\text { (dimensionless) }\end{array}$ & $\begin{array}{l}\text { 20 log } W_{4192} \\
\text { (decibels) }\end{array}$ & $\begin{array}{l}\text { Expanded (coverage } \\
\text { factor k=2) } \\
\text { uncertainty (decibels) }\end{array}$ \\
\hline 20000 & 1.0430 & 0.366 & 0.087 \\
\hline 19000 & 1.0317 & 0.271 & 0.073 \\
\hline 18000 & 1.0230 & 0.198 & 0.066 \\
\hline 17000 & 1.0161 & 0.139 & 0.063 \\
\hline 16000 & 1.0108 & 0.093 & 0.057 \\
\hline 15000 & 1.0065 & 0.056 & 0.052 \\
\hline 14000 & 1.0031 & 0.027 & 0.050 \\
\hline 13000 & 1.0007 & 0.006 & 0.046 \\
\hline 12000 & 0.9992 & -0.007 & 0.039 \\
\hline 11000 & 0.9995 & -0.004 & 0.031 \\
\hline
\end{tabular}




\section{UnCertainties of WAVE Motion CoRrection MeAsurement Results}

Expanded uncertainties of the measured values of $W_{4192}$ were estimated by applying recognized guidelines for evaluating and expressing uncertainties [9]. For these data, the combined standard uncertainty $u_{\mathrm{c}}$, all of the components of $u_{\mathrm{c}}$, and the expanded uncertainty (coverage factor $k=2$ ), are summarized as a function of frequency in Table 4. A Type A standard uncertainty, equal to the standard deviation of the eight values of $W_{4192}$ measured at each frequency, was calculated to indicate the repeatability of the results obtained with different microphone pairs. Several additional components, which are Type B standard uncertainties, were derived to account for the uncertainties in the values of the capillary tube correction, which was assumed to be equal to one $(0 \mathrm{~dB})$ regardless of the gas in the couplers, and the values of $f_{\mathrm{r}}^{\mathrm{h}}, \kappa^{\mathrm{a}}$ and $\kappa^{\mathrm{h}}$. These Type B standard uncertainties were derived by assuming a symmetric rectangular probability distribution, and estimating upper and lower bounds for the distribution. Given this assumption, the resulting standard uncertainty is equal to one-half the width of the distribution multiplied by a factor of $1 / \sqrt{ } 3$.

For the component due to the uncertainty in the capillary tube correction, distribution bounds were estimated by calculating capillary tube corrections according to Annex B of Ref. [4] and examining the effects of these corrections on the wave motion correction values. For the component arising from the uncertainty in the value of $f_{\mathrm{r}}^{\mathrm{h}}$, distribution bounds were estimated by determining the maximum and minimum frequencies measured for both hydrogen-filled couplers during the course of all the wave motion correction measurements. For the component due to the uncertainty in the value of $\kappa^{\mathrm{a}}$, distribution bounds were estimated based on slight differences found between the value calculated using Annex $\mathrm{F}$ of Ref. [4] and the value provided in Ref. [6]. For the component arising from the uncertainty in the value of $\kappa^{\mathrm{h}}$, the range between estimated bounds was increased over that used for air to allow for impurities in the hydrogen, water vapor, and air in the system. 
Table 4. Summary of uncertainties of the measured wave motion correction values.

\begin{tabular}{|c|c|c|c|c|c|c|c|c|}
\hline $\begin{array}{l}\text { Source of } \\
\text { uncertainty }\end{array}$ & $\begin{array}{l}\text { Standard } \\
\text { deviation } \\
\text { of results } \\
\text { for eight } \\
\text { microphone } \\
\text { pairs }\end{array}$ & $\begin{array}{l}\text { Capillary } \\
\text { tube } \\
\text { correction }\end{array}$ & $\begin{array}{l}\text { Hydrogen- } \\
\text { filled } \\
\text { cavity } \\
\text { resonance } \\
\text { frequency }\end{array}$ & $\begin{array}{l}\kappa \text { for } \\
\text { air }\end{array}$ & $\begin{array}{l}\kappa \text { for } \\
\text { hydrogen }\end{array}$ & \multirow[t]{3}{*}{$\begin{array}{l}\text { Combined } \\
\text { standard } \\
\text { uncertainty } \\
u_{\mathrm{c}}(\%)\end{array}$} & \multirow[t]{3}{*}{$\begin{array}{l}\text { Expanded } \\
\text { (coverage } \\
\text { factor } k=2 \text { ) } \\
\text { uncertainty } \\
2 u_{\mathrm{c}}(\%)\end{array}$} & \multirow{3}{*}{$\begin{array}{l}\text { Expanded } \\
\text { (coverage } \\
\text { factor } k=2) \\
\text { uncertainty } \\
\text { expressed as } \\
\text { the product } \\
\text { of } 20 \text { and the } \\
\text { log of } \\
{\left[1+\left(2 u_{\mathrm{C}} / 100\right)\right.} \\
(\mathrm{dB})\end{array}$} \\
\hline Type & $\mathrm{A}$ & $\mathrm{B}$ & $\mathrm{B}$ & B & $\mathrm{B}$ & & & \\
\hline $\begin{array}{l}\text { Frequency } \\
(\mathrm{Hz})\end{array}$ & \multicolumn{5}{|c|}{ Relative standard uncertainty (\%) } & & & \\
\hline 20000 & 0.450 & 0.030 & 0.190 & 0.040 & 0.097 & 0.501 & 1.002 & 0.087 \\
\hline 19000 & 0.381 & 0.065 & 0.131 & 0.040 & 0.097 & 0.421 & 0.842 & 0.073 \\
\hline 18000 & 0.340 & 0.093 & 0.108 & 0.040 & 0.097 & 0.383 & 0.766 & 0.066 \\
\hline 17000 & 0.324 & 0.103 & 0.072 & 0.039 & 0.097 & 0.363 & 0.726 & 0.063 \\
\hline 16000 & 0.289 & 0.106 & 0.054 & 0.039 & 0.096 & 0.329 & 0.658 & 0.057 \\
\hline 15000 & 0.252 & 0.117 & 0.050 & 0.039 & 0.096 & 0.301 & 0.602 & 0.052 \\
\hline 14000 & 0.235 & 0.130 & 0.022 & 0.039 & 0.096 & 0.289 & 0.578 & 0.050 \\
\hline 13000 & 0.199 & 0.139 & 0.020 & 0.039 & 0.096 & 0.265 & 0.530 & 0.046 \\
\hline 12000 & 0.153 & 0.129 & 0.008 & 0.039 & 0.096 & 0.226 & 0.452 & 0.039 \\
\hline 11000 & 0.118 & 0.086 & 0.003 & 0.039 & 0.096 & 0.179 & 0.358 & 0.031 \\
\hline 10000 & 0.135 & 0.009 & 0.003 & 0.041 & 0.100 & 0.173 & 0.346 & 0.030 \\
\hline 9000 & 0.101 & 0.007 & 0.003 & 0.041 & 0.100 & 0.148 & 0.296 & 0.026 \\
\hline 8000 & 0.081 & 0.009 & 0.003 & 0.041 & 0.100 & 0.135 & 0.270 & 0.023 \\
\hline 7000 & 0.073 & 0.012 & 0.028 & 0.041 & 0.100 & 0.134 & 0.268 & 0.023 \\
\hline 6000 & 0.077 & 0.007 & 0.024 & 0.041 & 0.100 & 0.135 & 0.270 & 0.023 \\
\hline 5000 & 0.062 & 0.024 & 0.030 & 0.040 & 0.100 & 0.130 & 0.260 & 0.023 \\
\hline 4000 & 0.026 & 0.045 & 0.011 & 0.040 & 0.099 & 0.119 & 0.238 & 0.021 \\
\hline 3000 & 0.044 & 0.039 & 0.005 & 0.040 & 0.099 & 0.122 & 0.244 & 0.021 \\
\hline 2500 & 0.051 & 0.029 & 0.005 & 0.040 & 0.099 & 0.122 & 0.244 & 0.021 \\
\hline 2000 & 0.079 & 0.013 & 0.008 & 0.040 & 0.098 & 0.133 & 0.266 & 0.023 \\
\hline 1500 & 0.066 & 0.016 & 0.007 & 0.040 & 0.097 & 0.125 & 0.250 & 0.022 \\
\hline 1000 & 0.093 & 0.067 & 0.002 & 0.040 & 0.096 & 0.155 & 0.310 & 0.027 \\
\hline
\end{tabular}




\section{REFERENCES}

[1] NIST Calibration Services Users Guide, Catalog of NIST Calibration Services, Mechanical, Acoustic Measurements, Pressure and Free-Field Responses of Microphones (25010C-25050C), NIST Special Publication 250, http://ts.nist.gov/MeasurementServices/Calibrations/acoustic.cfm\#25010c , [accessed November, 2008]

[2] IEC 61094-1-Ed. 2.0, Measurement Microphones - Part 1: Specifications for Laboratory Standard Microphones, International Electrotechnical Commission, Geneva, Switzerland (2000).

[3] ANSI S1.15-1997/Part 1 (R2006), American National Standard Measurement Microphones-Part 1: Specifications for Laboratory Standard Microphones, Acoustical Society of America, Melville, New York (1997).

[4] IEC 61094-2, Measurement Microphones - Part 2: Primary Method for Pressure Calibration of Laboratory Standard Microphones by the Reciprocity Technique, International Electrotechnical Commission, Geneva, Switzerland (1992).

[5] ANSI S1.15-2005/Part 2, American National Standard Measurement Microphones-Part 2: Primary Method for Pressure Calibration of Laboratory Standard Microphones by the Reciprocity Technique, Acoustical Society of America, Melville, New York (2005).

[6] ANSI S1.10-1966 (withdrawn), American National Standard Method for the Calibration of Microphones, American National Standards Institute, New York, New York (1997).

[7] IEC 61094-4, Measurement Microphones - Part 4: Specifications for Working Standard Microphones, International Electrotechnical Commission, Geneva, Switzerland (1995).

[8] W. Koidan, Hydrogen Retention System for Pressure Calibration of Microphones in Small Couplers, The Journal of the Acoustical Society of America, 35, No. 4, 614 (1963).

[9] B. N. Taylor and C. E. Kuyatt, Guidelines for Evaluating and Expressing the Uncertainty of NIST Measurement Results, NIST Technical Note 1297-1994 edition, U.S. Government Printing Office, Washington DC (1994). 